\title{
Plurilinguismo tra adattamenti, fraintendimenti, parodie, da Ruzante a Tarantino, nella scena italiana
}

\author{
Paolo Puppa \\ Università Ca’ Foscari, Dipartimento di Filosofia e beni culturali
}

Med različnimi viri izstopa Pirandellov roman iz leta 1902 Lontano z nekaterimi ibsenskimi vplivi, ki se osredotoča na različne, nasprotujoče si posledice, povezane z Babelovo milostjo in nesrečo. Gre za konstitutivno ambivalentnost vloge tujca med bedno parijo in skritim Bogom, ki se vrti okoli majhnega zvonika in nestrpnosti do tistih, ki govorijo drug jezik. Predstavljeni so emblematični liki v literarnem in gledališkem spominu o jezikovnem izgnanstvu, od mita o dobrem divjaku do Neznanca po Camusu, od Pinterjeve The Room do Tarantinove Stranieri.

Ključne besede: jezik v gledališču, Pirandello, Tarantino, večjezičnost, commedia dell'arte

Among the various sources, the Pirandellian novel of 1902, Lontano, with some Ibsenian influences, serves to focus on the different, contradictory implications relating to Babel's grace / misfortune. Constitutive ambivalence of the role of the foreigner, between miserable pariah and hidden God, in view of the small bell tower and intolerance towards those who speak a different language. Emblematic figures in the literary and theatrical memory about linguistic exile, from the myth of the good savage to the Stranger by Camus, from The room by Pinter to Stranieri by Tarantino.

Key words: language in theatre, Pirandello, Tarantino, multilingualism, commedia dell'arte

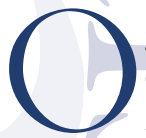

ggi, l'avvento di società multietniche-multiculturali e di un meticciato, in relazione ad un mercato globale e ad un'urbanizzazione espansa, si rovescia ne "l'apprestamento di difese e di meccanismi espulsivi a salvaguardia contro l'invasione del mondo 'altro"” (Domenichelli 1997, XVIV). Sempre più, di conseguenza, il concetto di straniero subisce spinte e controspinte contraddittorie, sino a divenire "stereotipo culturale, presente nella psicologia e nell'immaginario delle comunità umane /.../ fortemente implicato (e per questo rigido) nei processi di costruzione dell'identità dei popoli, delle comunità etniche e in quelle nazionali" (Ceserani 1997, 311). Pregiudizi reiterati si depositano così su questa figura, sottoposta nello stesso tempo ad accelerazioni e variazioni se- mantico-ideologiche. Del resto si tratta di dinamiche di lunga durata per un simile fantasma dell'immaginario, in qualche modo originario, che inizia già in natura, là dove l'animale marca il proprio territorio, pulsione presente pure nel mondo dell' infanzia ${ }^{1}$. Indubbia, in ogni caso, la potenzialità generativa nello straniero di costituirsi quale formidabile macchina narrativa, vedi Odisseo che incanta i Feaci e la dolce Nausicaa o Othello che seduce la bianca Desdemona grazie all'affabulazione enfatizzata delle loro peripezie. In un certo senso, la storia della cultura vede l'oscillare del valore positivo e negativo conferito a questa figura, alternanza legata altresì alla dialettica tra strada e casa, tra incontro coll'altro e

Bisogna aspettare, almeno in ambito italiano, Ceserani, Domenichelli e Fasano 2007, 2382-2391. 
chiusura all'altro, tra apertura al mondo e comunità chiusa. Si pensi da un lato alla febbre ardititesca-futurista poi confluita, sia pure sotto nuove vesti politiche, nel '68 e dall'altro al simbolismo pascoliano col mito struggente della demeure. Lo straniero che arriva da fuori pertanto risulta di volta in volta arricchimento educativo o all'opposto traumatica minaccia. Lacerazione che in fondo può essere ricondotta alla dicotomia antropologica e sociologica tra comunità migranti e sedentarie. E la paura che suscita tale fantasma è legata al fatto che lo straniero introduce virtualmente cambiamenti entro una società tradizionale. Ma tale oscillazione è implicita pure nei termini che connotano tale stereotipo. Non si dimentichi in effetti che hospes e hostis presentano la medesima radice nell' indoeuropeo (Benveniste $1976,272 \mathrm{e}$ sgg. $)^{2}$. E si può aggiungere che la detta coppia sprigioni una luce fosca, un $f a-$ scinus, nell'ambivalente accezione antica, sempre pronto a virare la disponibilità benevola nel suo opposto (Fasano 1997, LVIII). Nondimeno, se hostis indica insieme chi ospita e chi è ospitato, l'obbligo di ospitalità che spetta ad entrambi i due ruoli dischiude in tale semantema l'ipotesi di un dio travestito ${ }^{3}$. Virtualità che trascina con sé ovviamente implicazioni erotiche, come testimoniano Enea alla corte di Didone, Teseo a Creta, Giasone nella Colchide, Medea a Corinto, tutti in grado di suscitare amori travolgenti. Tant'è vero che la diffidenza verso lo straniero nutre e rimuove (non sempre) forti curiosità sessuali, da qui la fascinazione indubbia esercitata dallo stesso, per quel misto di attrazione e mitizzazione circa la libertà dei costumi in ambiti diversi rispetto al territorio ospitante. Basti citare il monologo, La nuit juste avant les forêts, $\mathrm{di}$ Bernard-Marie Koltès del 1977. Qui, il protagonista, un giovane straniero alla disperata ricerca di una stanza che lo ripari dalla notte sotto una pioggia devastante, nel suo ruminare appetiti e

Questo fa si che l'ospite e il nemico risultino uniti "dalla comune relazione di scambio e reciprocità” (Bodei 1997, 4).

Ad esempio, l'Odisseo omerico che ricorda nei suoi approdi a lidi sconosciuti "la sacralità dell'ospite straniero, sacro anche perché può celare un dio travestito" Marcialis $(1997,20)$. Per gli aspetti sinistri di tale stereotipo, cf. Perutelli (1997, 331-337). emarginazioni, desideri e umiliazioni subite, attraverso l'invocazione di un compagno, costituisce un perfetto simulacro di tutto ciò, declinato però quale fantasia di abiezione.

Nel trattamento negativo della figura, rientra di diritto L'étranger di Albert Camus, del '42, declinato in modo da far emergere una condizione di fatto anaffettiva con cui il personaggio osserva ad esempio la morte della madre, così come l'estraneità totale rispetto ai sentimenti e ai valori dagli altri da cui non si lascia condizionare, sino all'omicidio gratuito dell'arabo. Un'atonia che determina innanzitutto la lontananza da se stesso. In questo territorio entra di diritto, altresì, anche se su di un piano di minor assolutezza esistenziale e di un'autorevolezza letteraria inferiore, il moraviano Gli indifferenti del '29. Si potrebbe inquadrare una simile variante nel concetto freudiano di Unheimlichkeit, magari secondo lo schema di Julia Kristeva. Qui, lo straniero, in quanto sradicato e privo della identità originaria, viene fuori da noi stessi, facendo affiorare impulsi nascosti e debolezze (Kristeva 1988$)^{4}$. Rischiamo insomma di inabissarci nel cuore di tenebra del nostro io, con valenze conradiane. Come dimostra la storia di Dioniso nelle Baccanti euripidee, contrastato con furia compulsiva dal malcapitato Penteo, che si rifiuta di riconoscerlo in quanto Dio e si riduce poi a travestirsi in abiti muliebri per essere infine sbranato dalle donne, e dalla madre Agave per giunta. In compenso, lo straniero può essere valorizzato attraverso il mito del buon selvaggio, sulle orme di Montaigne (Fasano 1997, 463-48I; Greenblatt 1922) $)^{5}$, e in tal caso ci si sposta verso l'idealizzazione arcadico-utopica della natura, mentre avanza il relativismo, smantellando il postulato etnocentrico, in funzione di una nuova razionalità. Si costruiscono allora polarità quali primitivismo versus civiltà, stato di natura dei selvaggi senza governo e senza proprietà privata versus

4 La studiosa bulgara riprende il motivo dei processi psichici vissuti dall'emigrante anche sotto forma di thriller metafisico nel romanzo Meurtre à Byzance del 2004.

5 Del resto, anche la tradizione ebraica-cristiana universalizza la condizione dello straniero, in quanto ogni 'uomo sulla terra di Dio è solo di passaggio (Ceserani, Domenichelli e Fasano 2007, 23842385). 
gli ordinamenti sociali europei (Romeo 1989, 58 ; Fasano 1997, 470). Connessa a tale riabilitazione, ecco poi la prospettiva straniata che lo straniero consente ogni volta che si intende contestare l'esistente. L'illuminismo conosce spesso tale mascheratura, dalle Lettres persanes di Montesquieu del I72I ai severi foresti nella scena goldoniana. Così, la parlata levantina seriosa di Isidoro che condanna le pratiche femminili dispendiose ne Le done di casa soa del 1755, così il ricco Alì, disgustato dai castrati d'opera, ne L'impresario delle Smirne del 1759. Ritroviamo quest'occhio innocente e malizioso nell'orizzonte novecentesco, tra lo straniamento auspicato nella scena da Brecht e la categoria dell'ostranenie dei formalisti russi. Perché lo straniero, da sempre, è portatore di uno sguardo diverso e non integrato, alla lettera esotico. Se porta il caos nel micro mondo statico e chiuso in sé, questo sradicato e senza patria porta pure istanze di verità. Ci obbliga infatti a confrontarci colla percezione dell'altro, quasi a cogliere il proprio volto nell'occhio altrui, senza più le nostre abitudini, ossia gli automatismi narcisistici e auto protettivi. Quale variante, lo storico che si volge verso altri tempi sino a rischiare la nostalgia per il passato, come ammoniva Descartes ${ }^{6}$. Porsi fuori, ancora, per vedere meglio e vedere nuovo.

La demonizzazione dello straniero inizia, come si sa, dai Greci, capaci di inventare un lemma che idealmente comprendesse quanti non ne condividevano la loro etnicità. Questo, per essere precisi, dopo le invasioni persiane, come attesta la già citata Medea euripidea con tutta la sua minacciosa diversità. Il vocabolo escogitato, appunto $\beta \dot{\alpha} \rho \beta \alpha \rho \circ \varsigma$, rimanda ironicamente ad un'origine orientale e fa risuonare, per "onomatopea imitativa e reduplicativa” (Ceserani 1997, 319), chi si esprime in un linguaggio non articolato, insomma un parlante eterofono, di conseguen-

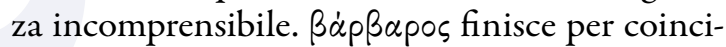
dere col non umano, coll'altro da sé con cui risul-

6 Per Descartes, tre figure dello straniero appaiono in tal senso, chi appunto assume attivamente lo sguardo dell'altro per mettere in crisi le proprie convinzioni, quindi la estraneità soggettiva, subita, dello sradicato, e infine quella dello storico. Nel Discours de la Méthode, è il filosofo dunque che si costituisce proprio in quanto assume uno sguardo straniante (Marcialis 1997, 19). ta impossibile entrare in relazione, condannabile pertanto nell'atto interlocutorio. Dunque, straniero, innanzitutto chi si esprime in una lingua diversa, vanificando in tal modo la fatica fatta da ognuno di noi per assimilare l'idioma materno, il vocabolario tanto vicino alla nostra animalità fisica, alle nostre radici. Opportuna, in questo snodo del discorso, la novella pirandelliana Lontano del 1902, in cui il marinaio norvegese Lars Cleen, che ha sposato Venerina, la ragazza siciliana di Porto Empedocle, si ritrova alla fine del racconto spaesato, specie nell'uso della parola. Una sorta di incubo, per cui rispetto agli altri esseri umani "si meravigliava poi nel veder loro battere le palpebre, com'egli le batteva, e muovere le labbra, com'egli le moveva. Ma che dicevano?" (Pirandello 1985, 957). Suoni del corpo, alla stessa stregua di quelli più fisiologici, e uguali, transculturali, e qui invece purtroppo ben differenziati. Perché lo straniero, se cerca di adattarsi, di assorbire il paese nuovo in cui si insedia, viene subito tradito e identificato nella sua diversità dall'orribile accento che svela l'appartenenza ad un'altra phoné. Pure Dante nel canto XVII, vv. 55-60 del Paradiso, esibisce tale esperienza in termini desolanti e depressivi: "Tu lascerai ogni cosa diletta”. Esilio quale autentica ordalia, prova drammatica che seleziona in termini darwiniani la categoria (Domenichelli 1997, XXXIV). Esprimersi fuori dalla propria origine comporta una lingua tagliata, deprivata, sradicata, lingua dell' infelicità, "lingua minorata" che però può rovesciarsi in "lingua della verità", "lingua filosofica” (Domenichelli 1997, XXXI). Questo, il primo trauma di chi è costretto a lasciare la terra madre. E' la tragedia di Babele, il passaggio in un al di là linguistico, di cui però un ebreo come George Steiner ci mostra i vantaggi, e insieme la fisiologica omologazione nei processi di comunicazione, verificata del resto nello scambio verbale coll'altro, entro il medesimo vocabolario (Steiner 1975). Una disgrazia e una grazia nello stesso tempo, così, l'atto di tradurre e di tradursi, riproponendo la medesima oscillazione di valore connessa al ruolo dello straniero. Naturalmente la dinamica non avviene mai alla pari, in quan- 
to ci sono lingue egemoniche e lingue subalterne, seguendo l'andamento dell'economia, la forza dell'esercito, e secondariamente della cultura. Così, uno svizzero, extracomunitario proprio come un marocchino qualsiasi, non viene mai nominato in tal modo.

Ebbene, portato in scena, lo straniero rivela subito la propria diversità meglio che sulla carta in quanto barbarismi fonici e solecismi espressivi vengono messi a nudo immediatamente. Di solito, la ribalta utilizza registri comici, ancora una volta facendo ridere di quel che si teme. Il caso di Angelo Beolco è esemplare in tal senso. Chiamato Ruzante dal nome del suo personaggio più celebre, Beolco si inquadra in un momento storico del teatro prima del teatro, in una fase laboratoriale in cui di fatto nulla appare definito. Non solo le poetiche o i pubblici, ma anche gli spazi e i tempi della rappresentazione non sono ancora canonizzati. Il periodo è quello della terza e quarta decade del Cinquecento, allorché si afferma e arriva al suo culmine la sua carriera. Al centro, il contadino, nella variante del brazente, colui cioè che porta le barche da terra, messo traumaticamente a contatto colla Storia, ovvero colla guerra. Il suo Parlamento allude proprio al terrore che incombe sulla Serenissima durante la Lega Santa e dopo la sconfitta di Agnadello, coi contadini costretti a inurbarsi, incalzati dall'impulso primario di scappare, di salvare la pelle. Arruolato a forza, inciampa così in altre lingue, in uno scenario angoscioso, sangue e morti dovunque, e una volta tornato a casa esibisce una irreversibile diversità. Basti rammentare l'insistenza con cui il reduce, sempre nel Parlamento, e nel suo grottesco colloquio col compare Menato, rimasto nei campi, infila di continuo una frase quasi apotropaica: 'S'a' fossè stò on' son stato io mi, a' no dissè cossí” (Ruzante 1967, 519)7, a marcare la frattura simbolica subita. Ma per l'attore autore pavano è proprio il plurilinguismo la nota espressiva caratterizzante, condivisa sia colla commedia letteraria rinascimentale sia collo spettacolo popolare del suo tempo. Si veda, per il

Questa è la prima emersione della domanda retorica, poi di continuo declinata dal personaggio secondo ambito, il buffone di tenda, ovvero la recita dietro un siparietto, molto richiesta nel menu delle feste, ossia il monologo dei buffoni ${ }^{8}$. In pratica, un ventriloquo in grado di imitare diverse parlate, una vera e propria drammaturgia aperta, disarticolata, non chiusa in un'opera determinata, ma finalizzata ad esaltarsi negli impromptus. Qui, l'attore mimetizzato dietro la cortina si limita a cambiar voce e lingua, creando un gioco virtuosistico di sdoppiamenti e di dissociazioni vertiginose, con un impatto irresistibile sul pubblico, e straordinari esiti comici, sempre specchio della grande paura provocata dai vuoti comunicativi tra idiomi l'un l'altro stranieri. E tuttavia tali sfasature vengono gestite in un clima festoso, spalmate democraticamente, senza interne gerarchie. Viene a cadere, in una parola, la koinè autoritaria e quaresimale, centripeta e normativa, soppiantata da quella centrifuga e carnevalesca ${ }^{9}$. Il motivo scardinante è quello dell'inversio ordinis, topica generativa nell'immaginario ruzantino, ovvero del roesso mondo, che rimanda al motivo del rovesciamento $\mathrm{e}$ dell'universalità di tale mutazione. L'attitudine anti-pedantesca di continuo insulta la lingua tecnica e la gergalità culta, magari tramite iper-correttismi e svarioni lessicali che fan precipitare un termine elatus nel territorio plebeius o rusticus. Frequenti risultano in tal senso le varietà idiomatiche nei copioni di Ruzante, basti osservare il Bilora, dove i dialetti si articolano con precisi riferimenti alle effettive parlate storiche, dal bergamasco aspro e quasi teutonico del servo, al pavano del protagonista e della sua donna, al veneziano cittadino del vecchio Andronico (di grande complessità come di solito i characters ruzantini, aperti e contraddittori prima del loro ri-

8 Si pensi a figure come Domenego Taiacalze o a Zuan Polo, da cui Ruzante preleva alcuni lazzi, come l'autoferirsi simulando l'aggressione, o l'uso reiterato dei soliloqui, come avviene spesso ne La Moscheta, l'opera più organica e riuscita di Beolco. Si tratta di arie, in termini di melodramma, da cui poi verranno attraverso un processo controverso i lazzi della commedia dell'arte. Sul genere e sui repertori del buffone di tenda (Vianello 2005, 87-I I7).

9 Ovviamente, il riferimento d'obbligo va agli studi di Michail. Bachtin sulle tipologie del comico carnevalesco, al suo canonico (edito dopo una travagliata gestazione solo nel 1965) F. Rabelais e la cultura popolare nel Medioevo, specie alle pp. 198-365 nell'edizione francese del 1970 (Puppa 1987, 149-179). 
dursi a fissità nelle maschere che ne derivano, come il senex libidinoso), destinato a morire sotto i colpi di Bilora stesso, ubriaco per prepararsi alla vendetta che dovrebbe restituirgli un risibile onore. Ne La Moscheta, ci imbattiamo in un quartetto di personaggi, oltre al villano prologhista ${ }^{\text {ro }}$ ad una vicina di scorcio. Il trio, inurbato nei sobborghi di Padova e costituito da $\mathrm{Ru}$ zante, Betía e dal loro compare Menato, legato da un disinvolto libertinismo rurale, viene completato dal soldato Tonin, con cui, specie i maschi, entrano in collisione. A sua volta il dualismo pavano-bergamasco attiva il consueto gioco di intarsi fonici, specie nei frequenti alterchi, anche professionali, tra contadino e soldato, tra reciproche accuse di "vilan" e di "megiolaro", ovvero di portaceste. E non si dimentichi che nel carattere di facchino, poi villano arruolato, si annidano indubbie premesse figurali del futuro Zanni degli scenari, ovviamente privato del côtè bulesco che ancora virilizza il personaggio ruzantino $^{\mathrm{I2}}$. Così pure le ripetute zuffe a distanza tra Ruzante e Tonin implicano scambi di accuse reciproche a base di "poltron" e "valent'omo" (Ruzante 1967, 649) ${ }^{13}$, consentendo altresì smottamenti etologici tra i due, con imitazioni sfasate e palinodie comportamentali. Per cui l'uomo d'armi calcola e simula una codardìa di comodo, pur di non trascendere e non pregiudicarsi il rapporto colla moglie del rivale. Dal canto suo, il contadino gioca a fare il "braoso", attratto dall'avversario, e si cimenta coll'asta in prove di aggressione da solo (come fa il collega Bilora, non fermandosi però alla simulazione) mentre vagheggia e minaccia un suo improvvisato arruolamento personale, nei modi e nelle forme a lui più convenienti. Ma, sempre ne La Moscheta, la di-

Io Mi riferisco al prologo a stampa, che differisce da quello marcianeo e veronese. Su La Moscheta, indispensabile la recente edizione critica a cura di D’Onghia 2010.

i Questo vale specialmente per la scena terza del quarto atto. Sul significato di megiolaro in quanto porta ceste Zorzi in Note a Ruzante $(1967,1372-1373$.)

I2 Sulla contrapposizione tra Ruzante e la commedia dell'arte, in garbata polemica colle tesi svalutative di Zorzi nei riguardi del sistema complessivo degli scenari Taviani (1985, 73-8I).

I3 Significativo il fatto che entrambi i personaggi sono convinti che l'altro di fatto non lavori. versificazione di accenti e idiomi si intensifica attraverso la mimesi ipercorretta, precipitata in macchina buffonesca, grazie alla recita, operata dal povero Ruzante, travestito nell'assemblaggio di ruoli forestieri, tra lo studente napoletano e il soldato di ventura spagnoleggiante ${ }^{\mathrm{I} 4}$, nella grande mouse trap del secondo atto. Qui il contadino, dietro il suggerimento dell'astuto Menato nei panni di un villico Iago, regista della scena meta teatrale, vuol saggiare la fedeltà coniugale della consorte. Alle prese con una lingua altra, per rendere lo studente "de la Talia, pulitàn" (Ruzante, 1967, 619), e poi il soldato smargiasso e spagnoleggiante, assembla iperboli e ridondanze relative alle forme pronominali "Sapítilo perché no me cognosseti lo io mi?", tra la collocazione a sproposito del suffisso "ano", la declinazione del verbo alla terza plurale, assente nel veneto, e il morfema "esse" a rendere la patina iberica. Da un lato allora "ben stàgano /.../ ve pàreno /.../ ve dégnano", e dall'altro "se volís essere las mias morosas, ve daranos de los dinaros" (Ruzante, 1967, 619). Salvo poi scoprire puntualmente la leggerezza della moglie, ben disposta ad accogliere in casa per denaro il foresto. Perché nella guerra le virtù domestiche non sono più moneta corrente e spendibile. Da notare che questa messinscena linguistica grazie agli spagnolismi pare prefigurare una delle future maschere in auge nella imminente commedia dell'arte, ossia il Matamoro nelle varie denominazioni. Menato che, rispetto alla coppia dei suoi protetti, può vantare una condizione economica meno assillata dal bisogno, in quanto proprietario di "buò, vache, cavale, piègore, puorçi e scroe” (Ruzante (1967, 591), nel quinto atto costruisce un altro play within play. Escogita l'incredibile spaesamento del malcapitato Ruzante, riuscendo ad allontanarlo da casa e poi a bastonarlo fino a tramortirlo, salvo poi organizzare una pace armata tra i contendenti e a godersi la donna. Ma la citata moltiplicazione degli idiomi in Ruzante ${ }^{15}$, relativa sia a cifre stilistiche che a vocabolari effet-

\footnotetext{
I4 Sulla confusione tra le due lingue Zorzi in Note a Ruzante (1967, I405).

is Utile, a tale proposito, Paccagnella 1998, I29-I 48.
} 
tivi ospitati nella commedia, consegna agli scenari frenetici delle maschere, egemoniche per almeno due secoli in Europa, la compresenza tra lessico alto e serioso degli Innamorati e il suo rovescio puntuale nel vocabolario fisiologico e triviale dei servi, ovvero uno scarto idiomatico e comportamentale tra due mondi. Il fatto è che da rispecchiamento del reale e della Storia (basti tenere a mente l'internazionalizzazione di una città come Venezia nel Rinascimento, simile alla New York o alla Londra di oggi), tale struttura si fa sempre più meccanismo codificato e astratto, macchina del comico e dell'equivoco. Inevitabile la riforma goldoniana, tesa a prosciugare via via il plurilinguismo nella sua riforma, dove però permangono tracce consistenti di equivoci e zuffe lessicali tra allofoni. Così opera ad esempio il finto armeno dell'Arlecchino burlesco ne La famiglia dell'antiquario del 1750 , che inventa un suo esotismo macaronico, quasi un involontario omaggio al gioco de La Moscheta, un arzigogolo al solito farcito di stilemi finto aulici che precipitano nello scatologico, per depredare il nobile Anselmo, collocato per prudenza nella lontana Palermo con una tecnica consolidata che rimanda agli ipercorrettismi deliranti nello schema Salomone-Marcolfo della memoria carnevalesca. La formazione dello Stato unitario e poi il Fascismo colla persecuzione delle compagnie dialettali completano tale opera, scardinando l'attore-autore in vernacolo grazie alla introduzione della regia e al privilegiare la drammaturgia in lingua. Spariscono così sia i copioni in dialetto, sia soprattutto le parlate multiple, la Babele democratica, lo straniero in scena. Questo per oltre un secolo. Poi, alla fine del secondo millennio, qualcosa si muove e preme per tornare all'antico, per riferirci al contesto italiano.

Nella produzione contemporanea, tale stratificazione inter-regionale si ritrova in Passione della torinese Laura Curino, datata 1995. Qui, nella storia del proprio apprendistato teatrale secondo la prospettiva dell'adolescente alle prese colla Storia grande ${ }^{16}$, la performer drammatur-

I6 Del resto, la stessa Curino mostra come la recente schiera dei narratori monologanti, da Paolini a Celestini, possegga precise ambizioni di scrittura, puntando spesso alla stampa dei loro copioni, ga riproduce per sobrie varianti foniche la varietà di accenti diversi, inghiottendo quasi in se stessa e poi rendendo con agilità irresistibile veneto e romagnolo, napoletano e toscano, pugliese e siciliano nella scena delle Supplenti. La Babele rispunta viceversa internazionale nella scrittura culta di un poeta che sembra richiedere più che mai l'oralità di una pronuncia teatrale. Alludo a Andrea Zanzotto il quale nel suo Filó, testo sollecitato come partitura sonora nel ' 76 per Casanova di Fellini, realizza forse meglio l'assunto di Luigi Meneghello (2002, 88-119) ${ }^{17}$, secondo cui il dialetto può, a volte, rendere nelle traduzioni anche i grandi testi tragici o romantici. Il montaggio filmico presenta all'inizio, assiepata a ridosso di uno stilizzato Ponte di Rialto, una folla assieme alle autorità e al doge, nel tipico interclassismo festivo della Serenissima. Si tratta però di un rito fascinoso e notturno, nell'etimo ambivalente del termine latino, ovvero misto di bellezza e di terrore, per l'apparizione di un'icona medusea che sorge dalle acque del Canal Grande. È questa la testa di un'orca enigmatica, gigantesca e nera, nume lagunare e madre mediterranea. Poi però si spezzano i pali, si strappano le funi, e il feticcio sparisce nelle acque, inabissandosi tra le vane orazioni dei presenti, da un lato implorazioni gementi e dall'altro imprecazioni beffarde e scatologiche. Zanzotto rispolvera per l'occasione il suo petél, ossia un idioma regressivo, il vecio parlar che alla ricerca di una glossia originaria mescola lalismo fiabesco, nenie arcaiche, sequenze tecnico-gergali, citazioni culte, in-

magari agevolati in ciò da una indubbia strategia editoriale, si pensi all'Einaudi pronta a pubblicare dvd dello spettacolo e copione. Ovviamente, si tratta più che mai di una formalizzazione dopo lo spettacolo, di cui fornisce un' eco riduttiva e in qualche modo provvisoria, nella dialettica tra scrittura oralizzante e oralità che si fa scrittura (Guccini 2005, 22-26) e anche, più in generale Stefanelli 2006. Perché tra testo scenico, oggi controllabile tramite registrazione elettronica, e testo a stampa si intrecciano di solito compromessi vari sul piano linguistico, di volta in volta orientati ad agevolare o ostacolare la ricezione di una sala allofona. Per il caso esemplare di Mistero Buffo di Fo, e la stratificazione successiva di inserti di varia estrazione dialettale rispetto al ceppo padano-veneto dell'esordio nel'69 (Barsotti 2007,209 e sgg.). Più in generale sulla mappatura del soliloquio teatrale Puppa 20 io.

I7 Per quanto riguarda la traduzione di passi dell'Hamlet di Sèspir Meneghello 2002, mentre per il Macbeth in Frammenti da Séspir in appendice del volumetto, I27-I29. 
trusioni materiche tardo poundiane e intuizioni junghiane sulla persistenza degli archetipi primari. Nel coro poliglotta, nella delirante polifonia che commenta la visione e la sua successiva dissolvenza, l'autore ha modo così di riversare un lessico inzuppato di latte e invaso dal fango, là dove l'io si annulla e con lui svaniscono le distinzioni spazio-temporali e la razionalità storicizzante. I suoni concitati, quasi il canto lamentoso intorno ad un parto bramato/temuto, paiono richiamare il sabba frenetico con cui Dario Fo, nel monologo plurale del Mistero buffo, presentava sette anni prima la sua Resurrezione di Lazzaro. Vi si riscontra, infatti, proprio il Carnevale, col vocabolario centrifugato, ancora una volta il babelico Fontego lagunare, il caos grottesco dell'evento numinoso rivissuto in chiave popolare, come se a dettare questo Vangelo apocrifo fosse lo sproloquio di uno Zanni ebbro e irrituale. In tale maniera, si conferma il recupero alto del dialetto in funzione dell'estro poetico, fuori da qualsiasi bozzettismo o intermezzo comico, mentre una scrittura più che mai preziosa si rovescia nell'estro e nel disordine vitalissimo di un corpo in scena.

Se il più importante uomo di teatro della scena occidentale, Shakespeare, inventaria quattro figure di straniero, ossia la donna, l'ebreo, il moro e il selvaggio (Deidda 1997, 497- 511 ${ }^{18}$, quello che qui interessa è l'ebreo dissociato tra un nomadismo incessante, quasi per il bisogno di "essere altrove", (Fink 1997, 139) e il tentativo reiterato di farsi assimilare in una determinata realtà culturale-linguistica. Straniero-ebreo-eretico-marginale diviene allora un volano per i motori dello sviluppo capitalistico moderno, assumendo sempre più rilievo nel suo decollo (Cotesta 2002, 9-I 4). È lui a saggiare l'effettiva apertura o meno delle società in cui cerca di penetrare, è lui a verificarne sulla sua pelle il grado di tolleranza: "Il senso di sicurezza o la paura verso l'altro sono l'espressione della fiducia che una comunità ha in se stessa. Se si crede nella propria capacità di integrare altri individui al pro-

I8 Deidda ricava tali categorie da Fiedler 1972, cui aggiunge le figure del malinconico e del mago, 497 . prio interno, si ha un atteggiamento di apertura verso lo straniero, non si teme la sua cultura" (Cotesta 2002, 5). Tanto più che parte del territorio degli Usa "è stata colonizzata da pellegrini inglesi alla ricerca di libertà religiosa”, così come lo sviluppo economico è stato realizzato "grazie anche a consistenti flussi di manodopera nera comprata sul mercato degli schiavi fiorente per secoli tra le due sponde dell'Atlantico" (Cotesta 2002, 3). Da un lato, pertanto, ipertrofia della razionalità, sprigionata "solo se si è estranei gli uni agli altri”, dall'altro la mobilità quale apertura all'esperienza (Cotesta 2002, I4). Ed è proprio l'ebreo a mostrarsi incapace di metter radici. E la sua estraneità si traduce nell'ambivalenza riguardo allo spazio, perché "la distanza nel rapporto significa che il soggetto vicino è lontano, mentre l'essere straniero significa che il soggetto lontano è vicino" (Cotesta 2002, I6). Essendo vicino-lontano, "non è riconosciuto come un individuo, ma come il membro di un tipo, di una classe, come l'elemento che è costantemente sospeso sul limite, che è fuori e di fronte" (Cotesta 2002, 2I). Una volta emigrato fuori, l'ebreo, il primo cosmopolita e cittadino del mondo, si emancipa dal suo vecchio habitat, e privo di legami com'è col nuovo mondo che l'accoglie anche con asprezza, non può, nella ricerca iniziale di assimilazione, che attivarsi lungo due traiettorie, ovvero "la secolarizzazione della società e l'individualizzazione dell'individuo" (Cotesta 2002, 25). Non basta, in quanto l'ebreo- straniero in quanto uomo marginale, secolarizzato e cosmopolita, è anche e soprattutto "individuo metropolitano", anticipando "le relazioni di estraneità esistenti tra gli uomini della modernità" (Cotesta 2002, 29).

Qui si aggancia la contraddizione oggettiva, o meglio la mimetizzazione operata in tal senso da Harold Pinter nel presentare la staticità esasperata del nucleo familistico in The room al debutto nel 1960, casa però mostrato quale bara-bunker e il condominio quale lager traslato. La stanza, vera protagonista del play, è proprio lo spazio vitale per sopravvivere rispetto al mondo esterno, pericoloso e violento, in uno scena- 
rio quasi da Day after. Tanto più viene accentuato il lato ornamentale e cosy del luogo, tanto più si infittiscono i segnali fuori della minaccia, quasi come nella polarità simbolica del dramma ibseniano tra salottino elegante e fiordo fuori, con apocalissi naturali che incombono sui personaggi ignari. Qui, l'ebraismo appare di sbieco, obliquamente, ${ }^{19}$ nel riferimento alla vecchia madre morta del padrone del caseggiato, in quanto "my mum was a Jewess" (Pinter 1968, 99). Al centro del copione, la coppia composta dalla sessantenne Rose Hudd e dal marito cinquantenne (sfasatura anagrafica significativa), Bert, camionista che esce anche nelle notti ghiacciate col suo van, personaggio muto per lo più tranne che nel furibondo finale, occupa l'appartamento coll'orgoglio di chi non fa che ribadire la propria tranquilla esistenza. Una vita regolata dal rito del tè, dalla cena preparata al marito, in un appagante ménage crepuscolare. In tale sclerotico e raggelante milieu, nessuno disturba ed è disturbato: "And we're not bothered. And nobody bothers us" (Pinter 1968, 93). E più avanti lo stesso concetto viene ribadito: "We're very quiet. We keep ourselves to ourselves. I never interfere. I mean, why should I? We've go tour room. We don't bother anyone else. That's the way it should be" (Pinter 1968, 105) ${ }^{20}$. Ma la quiete è ben presto incrinata dal sospetto che in basso ci sia un nuovo inquilino, "Maybe they're foreigners» (Pinter 1968,93$)$. Freddo fuori e dentro, e l'umidità che sale, scrostando e facendo cadere l'intonaco dal basement. Basta un battere alla porta per farli trasalire. L'antica paura dell'ebreo di cui parla Sholom Aleichem nel suo canonico dramma It's Hard To Be A Jew del i915. Il condomino però, veniamo informati, è pieno di inquilini "all sorts" (Pinter 1968, 99). Una seconda coppia, Mr e Mrs Sands, venuta a cercare dei locali, pretende che quello occupato dagli Hudd vada conside-

19 Harold Boom ha parlato, a proposito del suo teatro di una Shoah nascosta in Pinter (1987, I). Travestimenti e mascherature che ricordano un po' il film Lo squalo di Spielberg. Cf. pure Callimani 1985.

20 Poco oltre, a Mr Kidd è sempre Rose che insiste "I don't know anybody. We're quiet here. We've just moved into this district" (Pinter 1968, I I I). E infine, rivolta al cieco Riley, ribadisce disperata: "We're settled down here, cosy, quiet" (Pinter 1968, 113). rato libero, almeno secondo quanto riferito da una voce misteriosa del basement. Una autentica struggle for life pertanto si scatena, mentre ci si lamenta del buio che circola nell'intera casa. Poi arriva dalle viscere del caseggiato un cieco e negro, Riley, a parlarle di un padre che la rivuole, mentre lei non intende frequentare gente di "another district" (Pinter 1968, III). Emerge così una condizione di estraneità in se stessi, in chiave onirica. E intanto la lingua spezzata, paratattica di Pinter, scarnificata tra ellissi e spezzature, ruota attorno al rifiuto compulsivo della donna. L'incontro-scontro, segnato pure dal diverso nome che Riley le attribuisce, Sal e non Rose, sembra vanificare il processo di assimilazione e di integrazione operato dalla donna. Ad un tratto, per nulla motivato dalle battute precedenti, Rose si mette a toccargli gli occhi, la testa, le tempie, quasi a compiere una ricognizione corporale, un moto di identificazione o il ritorno inopinato di un antico desiderio. Nel brusco congedo del play, spunta Bert a parlare, descrivendo la corsa del suo van nella notte buia a gelata. Quindi si siede, davanti al negro, salvo poi con uno scatto imprevisto, farlo cadere e sbattergli più volte la testa sulla stufa (ricordo traslato delle camere a gas?). Rose intanto si copre gli occhi e mormora attonita tre volte "I can't see", come se fosse lei ora ad essere cieca. Infine, il blackout col sipario che cala sinistro, lasciando aperti tutti gli interrogativi.

Antonio Tarantino, nato nel 1938 , pertanto quasi coetaneo di Pinter nato nel ' 30 , arrivato tardi a teatro dalla pittura alla fine del secolo scorso, diviene presto il rappresentante di punta della nuova drammaturgia italiana, caratterizzata da una lingua bloccata su di una campionatura ossessiva di metafore ripetitive. Una gergalità da strada esalta il mondo dei clochards metropolitani, nella loro esibita, reciproca aggressività. E intanto vengono assurti a eroi del sottosuolo attraverso una parola impotente e onnipotente, che segna la loro laica via crucis. Si aggiunge, altresì, un costante sottotesto mitico, prelevato dalla tradizione evangelica, quale ombra grottesca dietro vicende proletarie. Una passionalità elemen- 
tare, con influssi vari, da Elsa Morante, a Sandro Penna, allo stesso Giovanni Testori, per l'insistita somiglianza tra questi paria umani e le figure della storia di Cristo. In Stranieri, inaugurato nel 2000, Tarantino riutilizza a modo suo The room. Stavolta è saltata la coppia, perché l'uomo, solitario vedovo, snocciola un paranoico monologo, di forte impronta bernhardiana. La lingua, seguendo i moduli cari al commediografo, impasta varie parlate, come la minaccia di ricorrere alle forze dell'ordine resa con "Polìs polìs/Polizei/Spazieren/Foera d'le bale" (Tarantino 2006, 2I). E questo flusso da blank verse viene spaziato verticalmente senza sintassi e punteggiatura, contrapposto alla prosa dei morti, salvo poi omologarsi al loro standard nel finale, non appena i due mondi vengono a collidere. Ma nella buia vicenda condominiale la estraneità è divenuta ormai intra-familiare, in quanto il vecchio sclerotico alle prese col catetere, e barricato dentro il proprio appartamento, simile ad una bara, non riconosce come parenti la moglie e il figlio tornati dall'al di là, a reclamare un contatto. All'inizio, l'uomo grida che non apre a nessuno, invitando a rivolgersi dalla vicina che "non paga il condominio" (Tarantino 2006, 17), dato che il "via via su e giù per le scale di sconosciuti" (Tarantino 2006 , 39), gli crea ansia. Li accusa altresì di parlare arabo, allertando difese contro il mondo esterno tipo "mettiamo che fuori dalla porta ci siano degli stranieri che vogliono entrare di prepotenza, magari per rapinarmi-ma io c'ho la cassaforte a muro" (Tarantino 2006, 50). E una volta "inchiodate le finestre blindato l'ingresso e le tapparelle-le tapparelle con tutti i chiavistelli che il fabbro mi ha messo su, io sono salvo" (Tarantino 2006, 39). Da Pinter, la bizzarra creatura preleva altresì il panico di essere schiodato dalla propria stanza-rifugio, perdendo magari "l'usu capione" (altro barbarismo lessicale), per cui "se loro fanno tanto a entrare si piazzano, aprono il frigo, si infilano nel mio letto, si mettono addosso la mia roba", e "e tu stai in un posto per trent'anni di fila, quel posto, alloggio o casa o giardino, diventa tuo" (Tarantino $2006,5 \mathrm{I}$ ). Ci informa pure del suo passato, confondendo però i tempi, declinandoli ancora al presente. Veniamo infatti a sapere che è stato commerciante di bilance affettatrici, da lui truccate nel peso, che si è presa la tessera socialdemocratica per opportunismo ideologico, che ha avuto un figlio, spesso picchiato forse per differenza culturale (il ragazzo s'è laureato in filosofia), e ancora che di notte si guarda le donne nude in televisione assicurando però di restare impassibile. In più, illustra il suo totale, disincantato antifamilismo contro il figlio fuggito a Roma, contro la nuora e la moglie, bella solo sul letto di morte, e la domestica straniera, magari incinta col fidanzato che gli beve i liquori. Una sorta di Alzheimer sentimentale e cognitivo scuote dalla fondamenta lo spazio della privacy. Nondimeno, questa mimesi sociologica di uno spaccato naturalistico nella realtà piccolo borghese del Nord Italia si tinge di colori neogotici, perché i congiunti (lo scopriamo a poco a poco) sono appunto defunti, stranieri nel senso metafisico della parola. Dialogo più che mai tra sordi, allora, gestito con ritmi vaudevilleschi, in una leggerezza a volte macabra, vedi ad esempio la descrizione del disfacimento dei corpi: "quello non ha neanche più le gengive e neppure più le labbra, e lasciamo pur stare l'aspetto generale della persona, che la dieta è la dieta, ma presentarsi così, nemmeno pelle e ossa, ma solo ossa" (Tarantino 2006, 34). A metà del montaggio però i due morti riescono a penetrare nel bunker, rivelandosi quali revenants venuti a portarlo via nel viaggio annichilente, refolo di una sonata di fantasmi strindberghiani, testardi e inflessibili. Quasi a prepararsi in tal senso, l'uomo si traveste da donna, si fa suo doppio (con qualche citazione da Orgia pasoliniana), e in quanto en travesti ne affetta la parlata, dizionario straniero che coniuga i verbi all'infinito, in una regressione culturale. Eccolo allora mormorare "Andare fuori/Essere bella giornata/ Se piovere/Noi europei/Avere ombrelli" (Tarantino 2006, 47-48), oppure "Mio marito/Essere uno bravo/Zone erogene nessuna/Zero/Che ci voleva l'indiano/Quello che col piffero/Fa uscire il serpente" (Tarantino 2006, 52), tra pesanti allusioni alla miseria sessuale della coppia. Una 
volta dentro la casa (il figlio aveva le chiavi!), la donna nota che il Padre "ci sta fissando" (Tarantino 2006,57 ), e che indossa il suo stesso abito. $\mathrm{Si}$ prova a calmarlo "Non è vero che gli stranieri ti vogliono uccidere", mentre lui ribatte "Pensavo addirittura che eravate due stranieri», mentre il figlio ribadisce che "noi non siamo stranieri". Intanto lo vestono, gli infilano la camicia, e lei gli toglie l'anello, nel ricordo pallido della Nora ibseniana per cui "siamo sciolti, per sempre" (Tarantino $2006,6 \mathrm{I}$ ). Ma il senso del tutto va ricercato nell'universalizzazione della estraneità che arriva fin dentro la casa, fin dentro noi stessi.

\section{Povzetek}

Na podlagi različnih virov iz stare in sodobne literature in gledališča prispevek analizira večjezičnost $v$ različnih oblikah uporabe pojavnosti ter posledice, ki se nanašajo na odnos med osebnostjo v nasprotju do drugega. Med različnimi viri izstopa Pirandellov roman iz leta 1902 Lontano z nekaterimi ibsenskimi vplivi, ki se osredotoča na različne, nasprotujoče si posledice, povezane z Babelovo milostjo in nesrečo. Gre za konstitutivno ambivalentnost vloge tujca med bedno parijo in skritim Bogom, ki se vrti okoli majhnega zvonika in nestrpnosti do tistih, ki govorijo drug jezik. Predstavljeni so emblematični liki v literarnem in gledališkem spominu o jezikovnem izgnanstvu, od mita o dobrem divjaku do Neznanca po Camusu, od Pinterjeve The Room do Tarantinove Stranieri. Judovska diaspora in njen mit o Babilonskem stolpu in igralčevi turneji sta dva različna načina razumevanja izleta $\mathrm{v}$ dežele $\mathrm{z}$ drugačnim jezikom. Končno se ta večjezičnost nanaša na tradicijo commedia dell'arte, v kateri tragedija zgodovine postane tudi komična igra.

\section{Summary}

Through different materials, taken from ancient and modern literature and theatre, this contribution analyzes the multiple language in its various uses and in the consequences referring to the self and the other. In particular, among the various sources, the Pirandellian novel of 1902, Lontano, with some Ibsenian influences, serves to focus on the different, contradictory implications relating to Babel's grace / misfortune. Constitutive ambivalence of the role of the foreigner, between miserable pariah and hidden God, in view of the small bell tower and intolerance towards those who speak a different language. Emblematic figures in the literary and theatrical memory about linguistic exile, from the myth of the good savage to the Stranger by Camus, from The room by Pinter to Stranieri by Tarantino. The Jewish diaspora and its myth of the Tower of Babel and the actor's tour are two different ways of understanding the excursion to lands with a different language. Finally this multilingualism refers to the tradition of the commedia dell'arte in which the tragedy of history also becomes a comic game.

\section{Riferimenti bibliografici}

Bakhtine, M. M. 1970. François Rabelais et la culture populaire au Moyen Age et sous la Renaissance, traduit par Andrée Robel. Paris: Gallimard.

Barsotti, A. 2007. Eduardo, Fo e l'attore-autore del Novecento. Roma: Bulzoni.

Benveniste, È. 1976. Il vocabolario delle istituzioni indoeuropee, vol. I: Economia, parentela, societá, tradotto da Mariantonia Liborio. Torino: Einaudi.

Bodei, R. 1997. "L'altro tra noi". In Lo straniero, a cura di Mario Domenichelli e Pino Fasano, vol. I, 3-I6. Roma: Bulzoni.

Calendoli, G. e G. Vellucci. 1987. Convegno internazionale di studi sul Ruzante (Padova 26-28 maggio 1983). Venezia: Corbo e Fiore.

Calimani, D. 1985. Radici sepolte. Il teatro di Harold Pinter. Firenze: Olschki.

Ceserani, R. 1997. "Sulle orme dello straniero:

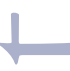

G

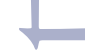

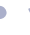

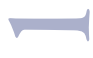

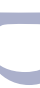
$\sqrt{20}$ frammenti di ricerca e problemi di metodo." In Lo straniero, a cura di Mario Domenichelli e Pino Fasano, vol. 2, 303330. Roma: Bulzoni.

Ceserani R., M. Domenichelli, e P. Fasano. 2007. Dizionario dei temi letterari, vol. 3. Torino: Utet.

Cotesta, V. 2002. Pluralismo culturale e immagini dell'Altro nella società globale. Roma-Bari: Laterza.

Curino, L. 1998. Passione. Novara: Interlinea. 
Deidda, A. 1997. “The Devil’s Part. Lo straniero nei drammi shakespeariani." In $L o$ straniero, a cura di Mario Domenichelli e Pino Fasano, vol. 2, 497- 51r. Roma: Bulzoni.

Domenichelli, M. 1997. "Pensiero straniero". In Lo straniero, a cura di Mario Domenichelli e Pino Fasano, vol. ı, XXV-XLIX. Roma: Bulzoni.

Domenichelli, M. e P. Fasano. 1997. Lo straniero. Atti del Convegno di Studi (I6-ID novembre 1994), voll. I e 2. Roma: Bulzoni.

Fasano, P. 1997. "Il racconto dello straniero." In Lo straniero, a cura di Mario Domenichelli e Pino Fasano, vol. I, LI-LXIX. Roma: Bulzoni.

Fasano, G. 1997. "Montaigne e il 'selvaggio': un apologia senza mito." In Lo straniero, a cura di Mario Domenichelli e Pino Fasano, vol. 2, 463-481. Roma: Bulzoni.

Fiedler, L. A. 1972. The Stranger in Shakespeare. New York: Stein and Day.

Fink, G. 1997. "Stranieri nella terra promessa: Israele nel romanzo ebraico-americano." In Lo straniero, a cura di Mario Domenichelli e Pino Fasano, vol. I, I35-I 47. Roma: Bulzoni.

Greenblatt, S. 1992. Marvellous Possessions: the Wonder of the New World. Chicago: University of Chicago Press.

Guccini, G., a cura di. 2005. La bottega dei narratori. Roma: Audino.

Kristeva, J. 1988. Étrangers à nous-mêmes. Paris: Fayard.

Kristeva, J. 2004. Meurtre à Byzance. Paris: Fayard.

Marcialis, M.T. 1997. “'...Quando si impiega troppo tempo a viaggiare, si diventa alla fine stranieri nel proprio paese'. Figure dell'estraneità da Platone a Benjamin." In Lo straniero, a cura di Mario Domenichelli e Pino Fasano, vol. I, I7-30. Roma: Bulzoni.

Meneghello, L. 2002. Trapianti: dall'inglese al vicentino. Milano: Rizzoli.

Paccagnella, I. 1998. "Il plurilinguismo di Ruzante." Quaderni veneti 27-28: I29-I 48.
Perutelli, A. 1997. "Il tema del perfidus hospes nella poesia erotica antica." In Lo straniero, a cura di Mario Domenichelli e Pino Fasano, vol. I, 331-337. Roma: Bulzoni.

Pinter, H. 1968. The Birthday Party. The Room. Two plays. New York: Grove Press.

Pirandello, L. 1985. Novelle per un anno, 2 vol. Milano: Mondadori.

Puppa, P. 1987. "Il contadino di Ruzante tra 'foire' carnevalesca e maschera sociale”. In Atti del Convegno internazionale di studi sul Ruzante (27-28-29 maggio 1987), a cura di Gianni Calendoli e Giacomo Vellucci, I 49-179. Venezia: Corbo e Fiore.

Puppa, P. 2010. La voce solitaria. Monologhi d'attore nella scena italiana tra vecchio e nuovo millennio. Roma: Bulzoni.

Romeo, R. 1989. Le scoperte americane nella coscienza italiana del Cinquecento. RomaBari: Laterza.

Ruzante. 1967. "Parlamento de Ruzante, che iera vegnú de campo". In Teatro, a cura di Ludovico Zorzi. Torino: Einaudi.

Ruzante. 20IO. Moschetta. Edizione critica e commento di S. D’Onghia. Venezia: Marsilio.

Stefanelli, S. 2006. Va in scena l'italiano. La lingua del teatro tra Ottocento e Novecento. Firenze: Cesati.

Steiner, G. 1975. After Babel: Aspects of Language and Translation. Oxford: Oxford University Press.

Tarantino, A. 2006. La casa di Ramallah e altre conversazioni. Milano: Ubulibri.

Taviani, F. 1985. "Una pagina sulla Commedia dell'Arte.” Quaderni di Teatro, 27: 73-8I.

Vianello, D. 2005. L'arte del buffone. Maschere e spettacolo tra Italia e Baviera nel XVI secolo. Roma: Bulzoni.

Zanzotto, A. 1999. "Filò". In Le poesie e le prose scelte, a cura di Stefano Del Bianco e Gian Mario Villalta, 46I-545. Milano: Mondadori.

Zorzi, L. 1967. Introduzione a Ruzante. In Teatro, a cura di Ludovico Zorzi, VIILXVII. Torino: Einaudi. 